\title{
Condições de vida do trabalhador docente: Associação entre estilo de vida e qualidade de vida no trabalho de professores de Educação Física
}

\author{
Worker's life conditions in the teaching profession: Association between \\ lifestyle and quality of life at work among physical education teachers
}

\author{
J. Both, J.V. Nascimento, C.N. Sonoo, C.A.F. Lemos, A.F. Borgatto
}

\begin{abstract}
O objetivo deste estudo foi de identificar o nível de correlação entre qualidade de vida no trabalho (QVT) e estilo de vida (EV) dos professores de Educação Física. A amostra de 1645 professores foi selecionada por meio de três estágios. O primeiro estratificou a região sul do Brasil conforme as unidades da federação, o segundo dividiu cada estado em mesoregiões e o terceiro considerou os Núcleos Regionais de Ensino como conglomerados. Na coleta de dados foram utilizados questionários que avaliaram a QVT e o EV. O teste Qui-quadrado para grupo único foi empregado para constatar discrepâncias de opiniões e comportamentos, e o teste de Spearman para avaliar o nível de correlações entre as variáveis investigadas, com um nível de significância de 5\%. Os resultados evidenciaram que os professores estão insatisfeitos com os salários, as condições de trabalho, a integração social e o tempo dedicado ao lazer, bem como possuem comportamentos negativos na alimentação, controle do estresse e atividade física. As evidências encontradas sobre o nível de associação entre os construtos dos parâmetros individual e sócio-ambiental das condições de vida do trabalhador docente em Educação Física foram consideradas insuficientes para indicar relação entre a QVT e o EV.

Palavras-chave: condições de vida, qualidade de vida no trabalho, estilo de vida, trabalho docente, educação física
\end{abstract}

ABSTRACT

The aim of this study was to identify the degree of correlation between quality of life at work (QVT) and personal lifestyle (EV) among physical education teachers. The sample, 1645 teachers, was selected in three phases. The first phase divided southern Brazil into federal states. The second subdivided each state into mesoregions and the third considered individual regional teaching centers. Data collection used questionnaires to evaluate QVT and EV. The single group Chi-square Test was used to establish discrepancies of opinion and behavior and the Spearman Test to evaluate correlations between the investigated variables, with a significance level of $5 \%$. The results showed that the teachers are unsatisfied with salaries, working conditions, social integration and available leisure time; also revealed poor diet and stress control and limited personal physical activity. Evidence to support an association between specific concerns and socio-environmental position was insufficient to establish correlations between QVT and EV.

Keywords: life conditions, quality of life at work, lifestyle, teaching profession, physical education

Submetido: 20.09.2009 | Aceite: 02.03.2010

Jorge Both. Programa de Pós-Graduação em Educação Física da Universidade Federal de Santa Catarina; Prefeitura Municipal de Florianópolis, Santa Catarina, Brasil.

Juarez Vieira do Nascimento e Adriano Ferreti Borgatto. Programa de Pós-Graduação em Educação Física da Universidade Federal de Santa Catarina, Brasil.

Christi Noriko Sonoo. Programa Associado de Pós-Graduação em Educação Física da Universidade Estadual de Maringá e Universidade Estadual de Londrina, Brasil.

Carlos Augusto Fogliarini Lemos. Curso de Educação Física da Universidade Regional Integrada do Alto Uruguai e das Missões - Campus de Santo Ângelo, Brasil.

Endereço para correspondência: Jorge Both, Rua Capitão Romualdo de Barros, 861 - Bloco 3 - Ap. 103 - Bairro

Carvoeira, Florianópolis - Santa Catarina, CEP: 88040-600 - Brasil

E-mail: jorgeboth@yahoo.com.br 
O profissional da educação desenvolve uma das atividades mais importantes da sociedade. Além de requerer desprendimento de tempo com a formação do estudante e o comprometimento com as tarefas de preparação das aulas, o trabalho docente compreende a realização de serviços administrativos da prática pedagógica, construção de planos e projetos escolares e avaliação das produções acadêmicas dos alunos. O desempenho destas atividades exige uma boa saúde física e mental por exigir grandes esforços físicos e psíquicos (Carlotto, 2002, 2004).

Nas investigações sobre as condições de vida do trabalhador docente, alguns estudos têm constatado que tanto problemas físicos, quanto o desgaste emocional vivenciados nos últimos anos são promotores do mal-estar docente (Brouwers \& Tomic, 2000; Dorman, 2003). Os afastamentos frequentes das atividades laborais de ensino estão associados às doenças do trabalho, as quais sofrem também interferência do estilo de vida individual (Bauer et al., 2006; Blair et al., 1984; Gasparini, Barreto, \& Assunção, 2005; Jacas, Castillo, Simón, \& Miclín, 2000; Porto et al., 2006; Reis et al., 2005, 2006; Santini \& Molina Neto, 2005; Vedovato \& Monteiro, 2008), bem como, dos baixos salários, da precariedade das condições de trabalho, da insatisfação geral com a educação e o baixo prestígio da profissão docente (Lapo \& Bueno, 2003).

Os professores de Educação Física, os quais trabalham conteúdos voltados à promoção da saúde na realidade escolar, têm relatado alguns problemas para adoção de um estilo de vida saudável (Nasário \& Shigunov, 2001). A depreciação do trabalho docente pode estar afetando o estilo de vida positivo que pode levar ao ressentimento, ou mesmo, ao afastamento da profissão (Santini \& Molina Neto, 2005).

Nesta perspectiva, acredita-se que aspectos inerentes à atividade docente, que revelam o nível de qualidade de vida no trabalho docente, bem como, aspectos associados ao estilo de vida de professores, podem interferir parcialmente ou diretamente na prática pedagógica do docente perante aos seus alunos e/ou a saúde deste trabalhador (Bauer et al., 2006; Blair et al., 1984; Unterbrink et al., 2007; Vedovato \& Monteiro, 2008).

Um aspecto a destacar é que o trabalho docente demanda tempo, desde a preparação da disciplina, das aulas e dos conteúdos abordados, bem como, do processo de avaliação (Carlotto, 2002, 2004). A realização destas atividades pode gerar sentimentos agradáveis e/ou desagradáveis, que dependem de vários fatores, como: a relação professoraluno, a relação professor-professor, a compensação recebida pelo trabalho, as condições para realizar o trabalho, as garantias legais em relação à sua ação docente, a estabilidade no emprego e possibilidade de progressão na carreira docente, a autonomia no trabalho, o tempo de lazer satisfatório, e o sentimento de importância da sua atuação profissional (Koustelios, 2005; Koustelios \& Tsigilis, 2005; Marques, Borges, \& Adorno, 2008; Hardman \& Marshall, 2000; Walton, 1973, 1974).

Atrelado a isto, destacam-se questões que estão associadas ao estilo de vida das pessoas, como: boa alimentação, ser ativo fisicamente, demonstrar comportamentos preventivos de saúde, cultivar bons relacionamentos e ter apenas um estresse que estimule a pessoa a realizar as suas atividades do dia-a-dia seria o ideal para um perfil de estilo de vida positivo (Bouchard et al., 1990; Heyward, 1998; Nahas, 2003; Nahas, Barros, \& Francalacci, 2000).

Portanto, sugere-se que uma boa percepção da qualidade de vida no trabalho docente, a qual é associada ao parâmetro sócio-ambiental, aliada a um perfil positivo de estilo de vida, que é vinculado ao parâmetro individual, pode ser um modelo de avaliação das condições de vida do trabalhador docente (Both \& Nascimento, 2009).

Assim, observando os escassos estudos que abordam a qualidade de vida no trabalho (parâmetro sócio-ambiental) e o estilo de vida 
(parâmetro individual) de docentes de Educação Física, e considerando a importância deste tipo de investigação para compreender melhor a percepção do trabalhador docente perante a sua função laboral, bem como diagnosticar os comportamentos que influenciam o estilo de vida de docentes, surgem os seguintes objetivos: a) identificar os comportamentos relacionados ao estilo de vida e as percepções sobre a qualidade de vida no trabalho dos professores de Educação Física; b) avaliar o nível de correlação interna dos constructos da qualidade de vida no trabalho e do estilo de vida; e, c) identificar o nível de correlação entre o estilo de vida e a qualidade de vida no trabalho dos professores de Educação Física que atuam nas redes estaduais de ensino que compõem a região sul do Brasil.

\section{MÉTODO}

A pesquisa descritiva exploratória, de corte transversal, teve como população os 13892 professores de Educação Física efetivos pertencentes aos quadros dos magistérios públicos estaduais das unidades da federação que compõem a região sul do Brasil.

\section{Amostra}

O processo de seleção da amostra ocorreu por meio de três estágios. $\mathrm{O}$ primeiro compreendeu a estratificação da região sul do Brasil conforme as unidades da federação. $\mathrm{O}$ segundo buscou estratificar cada estado em mesoregiões. E, o terceiro estágio considerou os Núcleos Regionais de Ensino (NREs) como conglomerado das mesoregiões dos seus respectivos estados.

O cálculo da amostra com uma margem de erro de $5.0 \%$, realizado inicialmente no programa computacional Epi-Info 3.5.1, estabeleceu uma amostra total de 374 professores. Entretanto, considerando que a taxa de retorno dos instrumentos foi de $28.69 \%$, a margem de erro amostral ficou estabelecida em $2.27 \%$, fixando o intervalo de confiança de $95 \%$.
Assim, a amostra ficou constituída por 1645 professores de Educação Física, sendo 654 docentes do estado do Paraná, 580 docentes do estado de Santa Catarina e 411 docentes do estado do Rio Grande do Sul. A média de idade dos professores era de 39.1 anos (desvio padrão de 8.8 anos) e, a média de tempo de docência era de 14.1 anos (desvio padrão de 8.9 anos).

Tabela 1

Descrição da amostra em relação aos aspectos sóciodemográficos e profissionais dos professores de Educação Física da região sul do Brasil

\begin{tabular}{|c|c|}
\hline Variáveis & $\%$ \\
\hline \multicolumn{2}{|l|}{ Sexo } \\
\hline Masculino & 43.0 \\
\hline Feminino & 57.0 \\
\hline \multicolumn{2}{|l|}{ Ciclos vitais } \\
\hline Até 29 anos & 16.7 \\
\hline 30 a 39 anos & 34.0 \\
\hline 40 a 49 anos & 36.3 \\
\hline 50 anos ou mais & 13.0 \\
\hline \multicolumn{2}{|l|}{ Estado civil } \\
\hline Casado & 62.8 \\
\hline Outro estado civil & 37.2 \\
\hline \multicolumn{2}{|l|}{$\begin{array}{l}\text { Ciclos de desenvolvimento profissional } \\
\text { (anos de docência) }\end{array}$} \\
\hline Entrada (0-4 anos) & 17.7 \\
\hline Consolidação (5-9 anos) & 18.7 \\
\hline Diversificação (10-19) & 32.8 \\
\hline Estabilização (20 anos ou mais) & 30.8 \\
\hline \multicolumn{2}{|l|}{ Formação académica } \\
\hline Graduado & 29.6 \\
\hline Pós-graduado & 70.4 \\
\hline \multicolumn{2}{|l|}{ Carga horária de trabalho semanal } \\
\hline Até 39 horas semanais & 45.2 \\
\hline 40 horas semanais ou mais & 54.8 \\
\hline \multicolumn{2}{|l|}{$\begin{array}{l}\text { Tempo de serviço no magistério público } \\
\text { estadual }\end{array}$} \\
\hline Até 3 anos de trabalho & 33.1 \\
\hline 4 a 11 anos de trabalho & 31.1 \\
\hline 12 a 19 anos de trabalho & 19.8 \\
\hline 20 anos de trabalho ou mais & 16.0 \\
\hline \multicolumn{2}{|l|}{ Pluriemprego } \\
\hline Não possui & 44.9 \\
\hline Possui & 55.1 \\
\hline
\end{tabular}


Os professores possuíam uma carga horária de trabalho semanal média de 32.1 horas/semana (desvio padrão de 10.6 horas/semana) e, possuíam vinculo empregatício com o magistério público do seu respectivo estado em média de 9.6 anos (desvio padrão de 8.2 anos). As demais variáveis sócio-demográficas e profissionais estão apresentadas na Tabela 1.

\section{Instrumentos}

$\mathrm{Na}$ coleta de dados foram utilizados dois instrumentos, além do questionário sóciodemográfico. O primeiro foi a Escala de Avaliação da Qualidade de Vida no Trabalho Percebida por Professores de Educação Física do Ensino Fundamental e Médio (QVT-PEF), o qual obteve uma excelente consistência interna (alpha de Cronbach de .95) (Both et al., 2006). O instrumento é constituído por 34 questões, as quais estão distribuídas nas seguintes oito dimensões, as quais compõem a matriz de análise da qualidade de vida no trabalho criada por Walton $(1973,1974)$.

Remuneração e compensação: É a relação entre o trabalho realizado e os fatores como: treinamento, salubridade, procura e oferta do serviço realizado pelo professor, salário médio estipulado pela comunidade e a capacidade da instituição mantenedora da escola de pagar o professor.

Condições de trabalho: Refere-se à exposição do professor a locais e/ou jornadas de trabalho indevidamente perigosos à saúde física e/ou psicológica.

Oportunidade imediata para uso $e$ desenvolvimento de capacidades humanas: $\mathrm{O}$ trabalho deve proporcionar a utilização dos conhecimentos e habilidades do professor, como também auxiliar no desenvolvimento de suas capacidades.

Oportunidade futura de crescimento e segurança: Relaciona-se às oportunidades do professor avançar e ter estabilidade na carreira.

Integração social na organização de trabalho: Está vinculada à natureza dos relacionamentos pessoais estabelecidos na instituição escolar.
Constitucionalismo na organização do trabalho: Compreende os direitos e deveres do professor perante o seu ambiente de trabalho.

Trabalho e espaço total de vida: É a relação de equilíbrio entre a dedicação pessoal com a vida profissional.

Relevância social da vida no trabalho: É a capacidade da instituição escolar de incutir no professor a importância do seu trabalho para a sociedade, como também, refere-se à seriedade e à responsabilidade social da instituição em relação aos serviços desempenhados.

O segundo instrumento foi o Perfil do Estilo de Vida Individual (PEVI) (Nahas et al., 2000), o qual obteve razoável consistência interna (alpha de Cronbach de .78) na avaliação do seu construto (Both et al., 2008). O questionário auto-administrado é composto por 15 questões divididas em cinco componentes: Alimentação, Atividade física, Comportamento preventivo, Relacionamentos e Controle do estresse.

\section{Procedimentos}

Para a realização da coleta de dados, inicialmente buscou-se autorização das três Secretarias Estaduais de Educação, para obter aprovação da investigação no Comitê de Pesquisa com seres humanos da Universidade Federal de Santa Catarina (036/07).

Após estes procedimentos, foram realizados contatos telefônicos com os NREs. Neste contato, buscava-se estabelecer a parceria na coleta das informações por meio do serviço de mala direta que os NREs possuem com cada escola de sua abrangência. Na sequência, foram encaminhados os pacotes aos NREs, contendo uma carta que informava o endereço para retorno dos questionários preenchidos, bem como, envelopes endereçados às escolas, os quais possuíam os dois instrumentos de coleta de dados, o questionário sócio-demográfico, e o termo de consentimento livre e esclarecido para serem preenchidos pelos participantes do estudo. $\mathrm{O}$ envelope encaminhado à escola também possuía uma carta explicativa ao diretor da escola sobre os procedimentos de 
retorno dos instrumentos preenchidos aos NREs. $\mathrm{Na}$ análise dos dados, utilizou-se inicialmente, as equações de ponderação de Lemos (2007) para categorizar as dimensões/componentes e a avaliação global da qualidade de vida no trabalho e do estilo de vida. Em seguida, como a amostragem utilizada não foi simples ao acaso, utilizou-se o inverso da probabilidade de seleção do indivíduo como peso para a utilização dos testes estatísticos. A estimação do peso do professor na amostra foi estipulada através da seguinte fórmula: $(\mathrm{Nr} / \mathrm{nar}) \times(\mathrm{nag} / \mathrm{Ng})$, onde: Nr: população regional; nar: amostra alcançada regional; nag: amostra alcançada geral; Ng: população geral.

\section{Análise estatística}

A análise estatística compreendeu a distribuição das frequências nas dimensões e componentes, bem como das avaliações globais da qualidade de vida no trabalho e do estilo de vida. Utilizou-se o teste Qui-quadrado para grupo único para constatar possíveis discrepâncias de opiniões no grupo de professores pesquisados. Destaca-se que o valor de referência neste teste estatístico foi de 33.3\%. O teste de Spearman foi empregado para analisar as correlações lineares entre as variáveis investigadas. Os testes estatísticos foram realizados no programa SPSS, versão 11.0, tendo como nível de significância de $5 \%$.

Os índices de correlação foram classificados conforme os pontos de corte sugeridos por Mitra e Lankford (1999), os quais estabelecem que correlações entre $.20 \quad$ e $\quad .40 \quad$ são consideradas como fracas, entre .40 e .60 são consideradas moderadas, e acima de .60 são consideradas como fortes.

\section{RESULTADOS}

Ao observar a distribuição das frequências das dimensões e da avaliação global da qualidade de vida no trabalho (Tabela 2), verificou-se que em todos os casos existiu uma tendência de opinião no grupo dos professores $(p<.001)$. Enquanto que a dimensão Remuneração e compensação demonstrou a maior frequência de professores insatisfeitos (54.0\%), as dimensões Condições de trabalho (39.8\%), Integração social na organização do trabalho (53.3\%) e Trabalho e espaço total de vida (46.1\%) demonstraram baixos índices de docentes satisfeitos. As várias dimensões demonstraram que a maioria dos professores de Educação Física está satisfeita com a qualidade de vida no trabalho (65.8\%).

Tabela 2

Distribuição das frequências das dimensões e da avaliação global da qualidade de vida no trabalho

\begin{tabular}{|c|c|c|c|c|}
\hline \multirow[b]{2}{*}{ Dimensões } & \multicolumn{3}{|c|}{ Qualidade de vida no trabalho } & \multirow[b]{2}{*}{$p^{*}$} \\
\hline & $\begin{array}{c}\text { Insatisfeitos } \\
(\%)\end{array}$ & $\begin{array}{l}\text { Indecisos } \\
(\%)\end{array}$ & $\begin{array}{l}\text { Satisfeitos } \\
(\%)\end{array}$ & \\
\hline Remuneração e compensação & 54.0 & 31.5 & 14.5 & $<.001$ \\
\hline Condições de trabalho & 29.6 & 30.6 & 39.8 & $<.001$ \\
\hline $\begin{array}{l}\text { Oportunidade imediata para uso e } \\
\text { desenvolvimento das capacidades humanas }\end{array}$ & 4.1 & 20.1 & 75.8 & $<.001$ \\
\hline $\begin{array}{l}\text { Oportunidade futura de crescimento e } \\
\text { segurança }\end{array}$ & 7.0 & 21.5 & 71.5 & $<.001$ \\
\hline Integração social na organização do trabalho & 10.6 & 36.1 & 53.3 & $<.001$ \\
\hline $\begin{array}{l}\text { Constitucionalismo na organização do } \\
\text { trabalho }\end{array}$ & 5.5 & 16.7 & 77.8 & $<.001$ \\
\hline Trabalho e espaço total de vida & 23.0 & 30.9 & 46.1 & $<.001$ \\
\hline Relevância social da vida no trabalho & 4.1 & 16.4 & 79.5 & $<.001$ \\
\hline $\begin{array}{l}\text { Avaliação global da qualidade de vida no } \\
\text { trabalho }\end{array}$ & 8.2 & 26.0 & 65.8 & $<.001$ \\
\hline
\end{tabular}


Tabela 3

Distribuição das frequências dos componentes e da avaliação global do estilo de vida

\begin{tabular}{lcccc}
\hline \multirow{2}{*}{ Dimensões } & \multicolumn{3}{c}{ Estilo de vida } & \multirow{2}{*}{$p^{*}$} \\
\cline { 2 - 4 } & $\begin{array}{c}\text { Negativo } \\
(\%)\end{array}$ & $\begin{array}{c}\text { Intermediário } \\
(\%)\end{array}$ & $\begin{array}{c}\text { Positivo } \\
(\%)\end{array}$ & $<.001$ \\
\hline Alimentação & 29.5 & 32.1 & 38.4 & $<.001$ \\
Atividade física & 19.2 & 22.6 & 58.2 & $<.001$ \\
Comportamento preventivo & 7.5 & 12.2 & 80.3 & $<.001$ \\
Relacionamentos & 5.6 & 14.9 & 79.5 & $<.001$ \\
Controle do estresse & 21.1 & 27.2 & 51.7 & $<.001$ \\
Avaliação global do estilo de vida & 6.0 & 27.1 & 66.9 & $<$ \\
* Valor de $p$ estimado através do Qui-quadrado para grupo único (referência das proporções $=33.3 \%)$ &
\end{tabular}

* Valor de $p$ estimado através do Qui-quadrado para grupo único (referência das proporções $=33.3 \%$ )

Em relação à distribuição das frequências dos componentes e da avaliação global do estilo de vida (Tabela 3), observou-se que em todos os casos os professores de Educação Física demonstraram uma tendência de comportamento positivo $(p<.001)$. No entanto, os professores investigados evidenciaram comportamentos positivos menos representativos nos componentes Alimentação (38.4\%), Atividade física (58.2\%) e Controle do estresse (51.7\%).

Nos componentes Comportamento preventivo (80.3\%) e Relacionamentos $(79.5 \%)$ constatouse os melhores índices de comportamento positivo. Ao considerar os valores encontrados em todos os componentes, observou-se que $66.9 \%$ dos docentes possuem comportamento positivo na avaliação global do estilo de vida (Tabela 3).

Na Tabela 4 são apresentadas as associações entre as dimensões e avaliação global da qualidade de vida no trabalho. Os resultados revelaram que a maioria das dimensões obteve uma forte correlação com a avaliação global da qualidade de vida no trabalho $\left(r_{\mathrm{s}} \geq .60\right)$, exceto para a Remuneração e compensação $(r=.41) \mathrm{e}$ Trabalho e espaço total de vida $(r=.46)$, onde demonstraram moderada correlação.

Tabela 4

Distribuição das frequências dos componentes e da avaliação global do estilo de vida

\begin{tabular}{ccccccccc}
\hline Variáveis & QVT1 & QVT2 & QVT3 & QVT4 & QVT5 & QVT6 & QVT7 & QVT8 \\
\hline QVT1 & - & - & - & - & - & - & - & - \\
QVT2 & $.32^{*}$ & - & - & - & - & - & - & - \\
QVT3 & $.27^{*}$ & $.49^{*}$ & - & - & - & - & - & - \\
QVT4 & $.22^{*}$ & $.44^{*}$ & $.46^{*}$ & - & - & - & - & - \\
QVT5 & $.23^{*}$ & $.42^{*}$ & $.47^{*}$ & $.38^{*}$ & - & - & - & - \\
QVT6 & $.19^{*}$ & $.40^{*}$ & $.51^{*}$ & $.41^{*}$ & $.51^{*}$ & - & - & - \\
QVT7 & $.19^{*}$ & $.30^{*}$ & $.29^{*}$ & $.21^{*}$ & $.22^{*}$ & $.21^{*}$ & - & - \\
QVT8 & $.18^{*}$ & $.41^{*}$ & $.52^{*}$ & $.49^{*}$ & $.44^{*}$ & $.48^{*}$ & $.23^{*}$ & - \\
QVT Geral & $.41^{*}$ & $.64^{*}$ & $.70^{*}$ & $.60^{*}$ & $.64^{*}$ & $.60^{*}$ & $.46^{*}$ & $.62^{*}$ \\
\hline
\end{tabular}

Legenda: QVT1: Remuneração e compensação; QVT2: Condições de trabalho; QVT3: Oportunidade imediata para uso e desenvolvimento das capacidades humanas; QVT4: Oportunidade futura de crescimento e segurança; QVT5: Integração social na organização do trabalho; QVT6: Constitucionalismo na organização do trabalho; QVT7: Trabalho e espaço total de vida; QVT8: Relevância social da vida no trabalho; QVT Geral: Avaliação global da qualidade de vida no trabalho; * $p<.001$ 
A análise geral das associações entre as dimensões desta variável revelou um maior número de correlações moderadas (14 associações) e fracas (11 associações), sendo encontradas valores muito fracos de correlação em apenas três cruzamentos de dimensões. As dimensões que apresentaram maiores índices de correlações com as demais foram as Condições de trabalho e Oportunidade imediata para uso e desenvolvimento das capacidades humanas. As Condições de trabalho demonstraram fraca associação com a Remuneração e compensação e Trabalho e espaço total de vida, e moderada correlação com as demais dimensões. Resultados similares das associações foram encontrados na dimensão Oportunidade imediata para uso e desenvolvimento das capacidades humanas.

No que diz respeito às correlações entre os componentes e a avaliação global do estilo de vida (Tabela 5), constatou-se que apenas a avaliação global do estilo de vida e o componente Atividade física apresentou forte correlação $(r=.61)$. Os demais componentes evidenciaram moderada correlação com a avaliação global do estilo de vida $\left(r_{s}\right.$ entre $.41 \mathrm{e}$ .59). As associações entre os restantes componentes do construto do estilo de vida evidenciaram fracas ou muito fracas correlações, onde os componentes
Comportamento preventivo e Relacionamentos apresentaram os menores valores de correlação.

Tabela 5

Distribuição das frequências dos componentes e da avaliação global do estilo de vida

\begin{tabular}{cccccc}
\hline Variáveis & PEVI1 & PEVI2 & PEVI3 & PEVI4 & PEVI5 \\
\hline PEVI1 & - & - & - & - & - \\
PEVI2 & $.26^{*}$ & - & - & - & - \\
PEVI3 & $.21^{*}$ & $.16^{*}$ & - & - & - \\
PEVI4 & $.09^{*}$ & $.24^{*}$ & $.11^{*}$ & - & - \\
PEVI5 & $.27^{*}$ & $.28^{*}$ & $.17^{*}$ & $.28^{*}$ & - \\
PEVI Geral & $.54^{*}$ & $.61^{*}$ & $.41^{*}$ & $.42^{*}$ & $.59^{*}$ \\
\hline
\end{tabular}

Legenda: PEVI1: Alimentação; PEVI2: Atividade física; PEVI3: Comportamento preventivo; PEVI4:

Relacionamentos; PEVI5: Controle do estresse; PEVI

Geral: Avaliação global do estilo de vida; * $p<.001$

$\mathrm{Na}$ tentativa de identificar o nível de associação dos construtos qualidade de vida no trabalho e estilo de vida (Tabela 6), constatouse que a maioria dos cruzamentos das dimensões, bem como das avaliações globais de ambos os construtos demonstraram correlação muito fraca ( $r_{s}$ abaixo de .20). Destaca-se que a associação entre a avaliação globais da qualidade de vida no trabalho e do estilo de vida somente apresentou um índice de correlação de $r=.09$.

Tabela 6

Associações das correlações entre as dimensões e avaliação global da qualidade de vida no trabalho com os componentes e a avaliação global do estilo de vida

\begin{tabular}{cccccccccc}
\hline Variáveis & QVT1 & QVT2 & QVT3 & QVT4 & QVT5 & QVT6 & QVT7 & QVT 8 & $\begin{array}{c}\text { QVT } \\
\text { Geral }\end{array}$ \\
\hline PEVI1 & .03 & $.06^{*}$ & $.06^{*}$ & .05 & $.06^{*}$ & .04 & .05 & $.06^{*}$ & $.07^{*}$ \\
PEVI2 & .01 & $.08^{* *}$ & $.07^{*}$ & $.06^{*}$ & $.06^{*}$ & .02 & $.13^{* *}$ & $.06^{*}$ & $.08^{* *}$ \\
PEVI3 & -.01 & .02 & .02 & $.08^{* *}$ & .05 & -.01 & -.01 & .03 & .02 \\
PEVI4 & $.09^{* *}$ & $.09^{* *}$ & $.14^{* *}$ & $.09^{* *}$ & $.11^{* *}$ & $.07^{*}$ & $.15^{* *}$ & $.11^{* *}$ & $.14^{* *}$ \\
PEVI5 & $.11^{* *}$ & $.16^{* *}$ & $.13^{* *}$ & $.08^{* *}$ & $.09^{* *}$ & $.08^{* *}$ & $.27^{* *}$ & $.10^{* *}$ & $.17^{* *}$ \\
PEVI Geral & $.07^{*}$ & $.09^{* *}$ & $.09^{* *}$ & $.08^{* *}$ & $.07^{*}$ & .04 & $.16^{* *}$ & $.08^{* *}$ & $.09^{* *}$ \\
\hline
\end{tabular}

Legenda: PEVI1: Alimentação; PEVI2: Atividade física; PEVI3: Comportamento preventivo; PEVI4: Relacionamentos; PEVI5: Controle do estresse; PEVI Geral: Avaliação global do estilo de vida; QVT1: Remuneração e compensação; QVT2: Condições de trabalho; QVT3: Oportunidade imediata para uso e desenvolvimento das capacidades humanas; QVT4: Oportunidade futura de crescimento e segurança; QVT5: Integração social na organização do trabalho; QVT6: Constitucionalismo na organização do trabalho; QVT7: Trabalho e espaço total de vida; QVT8: Relevância social da vida no trabalho; QVT Geral: Avaliação global da qualidade de vida no trabalho; ${ }^{*} p \leq .01,{ }^{* *} p \leq .001$ 
O maior índice de correlação encontrada foi entre a dimensão Trabalho e espaço total de vida que compõe a qualidade de vida no trabalho e o componente Controle do estresse que faz parte do construto do estilo de vida, embora seja considerada uma fraca correlação $(r=.27)$, tendo em conta os critérios de Mitra e Lankford (1999).

\section{DISCUSSÃO}

As evidências encontradas revelaram que os professores de Educação Física estão insatisfeitos principalmente com os salários. A remuneração adequada constitui uma reivindicação antiga do professorado de vários paises, como a Malásia (Jabnoum \& Fook, 2001), Indonésia (Nilan, 2003), Brasil (Lapo \& Bueno, 2003; Molina Neto, 1998), Grécia (Koustelios, 2001) e Venezuela (Viera, Hernandez, \& Fernández, 2007). Andrews (1993) e Nilan (2003) destacam que em muitos casos, os professores acumulam uma carga horária semanal de trabalho superior a 40 horas, em dois ou mais postos de trabalho, para ter uma remuneração digna que satisfaça suas necessidades pessoais e familiares.

Sobre as condições de trabalho, observou-se que a maioria dos docentes está satisfeita. Entretanto, observa-se que há altos índices de professores indecisos e insatisfeitos. Similar ao descontentamento com os salários, docentes de diferentes nacionalidades (indonésios, venezuelanos, brasileiros e gregos) também relataram grande insatisfação com relação aos aspectos materiais disponibilizados à intervenção docente (Koustelios, 2001; Lapo \& Bueno, 2003; Nilan, 2003; Viera et al., 2007). Na realidade brasileira, há discrepâncias sobre este aspecto que estão associadas às diferentes políticas públicas implementadas nas unidades federativas. Em alguns casos, as instituições escolares recebem auxilio das associações de pais e mestres, da comunidade próxima à escola, das direções escolares, ou mesmo, do processo de renovação das instalações das escolas promovida pelas instituições públicas responsáveis pela gestão escolar.
Os resultados que evidenciaram os baixos índices de satisfação dos professores investigados nas dimensões que se referem à integração social dos docentes no ambiente de trabalho e o equilíbrio do tempo dedicado ao lazer e ao trabalho, foram similares aos encontrados com docentes da Austrália (MacDonald, 1995), Singapura (Smith \& Leng, 2003), Israel (Fejgin, Talmor, \& Erlich, 2005) e Brasil (Molina Neto, 1998; Santini \& Molina Neto, 2005). Além de demonstrarem novos indicadores de insatisfação docente no ambiente escolar, os resultados deste estudo apontam para a necessidade de rever não somente as políticas públicas implementadas pelas Secretárias Estaduais de Educação, mas também, para a modificação de condutas inadequadas frente aos docentes realizadas pelas direções escolares, equipes pedagógicas e o restante da comunidade escolar.

Um aspecto a destacar são os valores positivos demonstrados nas dimensões que tratam sobre a autonomia do trabalho pedagógico, oportunidade de crescimento e segurança, legislação do trabalho docente e a relevância do trabalho desempenhado, o que fez com que a cada dez professores, seis docentes demonstraram estar contentes com a avaliação global da qualidade de vida no trabalho.

No que diz respeito à análise do estilo de vida, as evidências encontradas revelaram que os componentes Alimentação e Controle do estresse são os comportamentos mais preocupantes dos professores investigados. De fato, a percepção de sobrecarga de trabalho no final do ano, os problemas com os alunos, a baixa remuneração, e a dificuldade de manter uma alimentação equilibrada e adequada são alguns fatores que podem aumentar o estresse e comprometer uma alimentação saudável dos docentes (Al-Mohannadi \& Capel, 2007; Nahas et al., 2000).

Em relação ao Controle do estresse, destaca-se que a sobrecarga de trabalho, atrelada às obrigações familiares e da comunidade, bem como às características pessoais e profissionais 
(sexo, faixa etária, formação acadêmica, e tempo de serviço) podem interferir na forma que os docentes enfrentam os fatores estressantes (Al-Mohannadi \& Capel, 2007; Vedovato \& Monteiro, 2008). Os problemas relacionados ao estresse docente, além de afetarem o nível de integração do docente no ambiente de trabalho, tem acarretado problemas patológicos, como a síndrome de burnout, que pode afastar o trabalhador docente parcialmente ou totalmente das suas funções laborais (Brouwers \& Tomic, 2000; Dorman, 2003; Gasparini, Barreto, \& Assunção, 2005; Pillay, Goddard, \& Wilss, 2005; Santini \& Molina Neto, 2005; Unterbrink et al., 2007).

Os resultados da investigação apontaram valores moderados de comportamento positivo no componente Atividade física, sendo que seis em cada dez docentes relatou comportamento satisfatório. Esta constatação é preocupante, pelo fato de que os professores de Educação Física são frequentemente responsabilizados pela docência de conteúdos voltados a atividade física no ambiente escolar. Alguns docentes investigados podem não estar demonstrando na prática os seus conhecimentos, o que pode resultar ao descrédito do docente perante a sua atividade pedagógica (Nasário \& Shigunov, 2001).

Os altos índices de comportamentos positivos foram encontrados nos componentes Comportamento preventivo e Relacionamentos, os quais auxiliaram positivamente na avaliação global do estilo de vida. Nesta perspectiva, os resultados demonstraram que a cada dez docentes, seis possuíam comportamentos saudáveis.

Apesar dos valores percentuais dos componentes/dimensões e das avaliações globais da qualidade de vida no trabalho e estilo de vida possam ser similares, os índices de correlação encontrados foram considerados muito fracos. Além de indicarem a baixa associação entre os parâmetros individual e sócio-ambiental das condições de vida do trabalhador docente, os professores demonstraram diferentes tipos de comportamento do seu estilo de vida ao mesmo tempo em que percebem a sua qualidade de vida no trabalho docente.

A análise pormenorizada do construto estilo de vida apontou que a maioria das correlações entre os componentes foi considerada fraca ou muito fraca. Tais valores podem estar associados à abrangência da temática "estilo de vida", a qual avalia componentes mais heterogêneos (Both et al., 2008). Por outro lado, observou-se na avaliação do construto da qualidade de vida no trabalho, índices de correlação superiores aos encontrados na análise do construto do estilo de vida. Neste caso, a maioria das correlações entre as dimensões foi classificada de fraca a moderada, revelando maior proximidade dos assuntos abordados entre as dimensões da matriz analítica centrada no "trabalho" (Walton, 1973, 1974).

Ao analisar as associações entre as matrizes de analise da qualidade de vida no trabalho e do estilo de vida, constatou-se fraca correlação entre os indicadores de controle do estresse e de equilíbrio de tempo para o trabalho e para o lazer. O que pode ser resultante da possível relação de causalidade com a patologia estresse, conforme apontam os estudos de Carlotto (2002, 2004) e Vedovato e Monteiro (2008). Nas demais associações detectou-se que as dimensões relacionadas à qualidade de vida no trabalho interferem muito pouco nos componentes que compõem o construto do estilo de vida. Contudo, tais diferenças podem ser um fator positivo na avaliação das condições de vida do trabalhador docente, pelo fato de poder analisar diferentes perspectivas, de forma abrangente, da vida do professor.

Destacam-se, como limitações deste estudo, dois fatores que podem interferir na análise dos dados. O primeiro é a percepção que os docentes possuem em relação à qualidade de vida no trabalho. Nesta perspectiva um mesmo fato pode ter respostas diferentes em um grupo de pessoas, não tendo certo ou errado, mas sim, opiniões divergentes sobre o mesmo tema 
(Hardman \& Marshall, 2000). Diferente do estilo de vida, onde se avalia o comportamento, o qual pode ser negativo, intermediário ou positivo.

O segundo é referente aos diferentes planos de carreira dos docentes de Educação Física dos magistérios públicos estaduais, os quais podem interferir na análise dos valores percentuais do grupo. Considerando este raciocínio, um estado pode ter uma tendência positiva para os comportamentos do estilo de vida e de percepção de satisfação para o trabalho, enquanto que outra unidade da federação pode possuir uma tendência negativa no comportamento do estilo de vida e de insatisfação no trabalho, o que pode evidenciar uma heterogeneidade do grupo. Entretanto, as análises das correlações não são prejudicadas, pois as mesmas têm como lógica para a construção do índice de correlação a individualidade de cada professor que compõe a amostra.

\section{CONCLUSÕES}

Os resultados obtidos neste estudo permitem concluir que os professores de Educação Física, embora satisfeitos com a avaliação global da qualidade de vida no trabalho, estão descontentes principalmente com a remuneração. Além disso, os baixos níveis de satisfação sobre as condições de trabalho, o tempo equilibrado entre lazer e trabalho e integração social na comunidade escolar são assuntos preocupantes na percepção dos docentes investigados, refletindo aspectos macro organizacionais ligados às Secretarias de Educação de cada estado, como também, de aspectos micro organizacionais vinculados às direções das unidades escolares.

No que se refere ao estilo de vida, constatou-se que a maioria dos professores possui comportamentos positivos na avaliação global, apesar de alguns docentes relatarem índices negativos e intermediários sobre o comportamento alimentar, atividade física e controle do estresse. A implantação de políticas institucionais e ações de formação continuada são recomendadas, tanto para contemplar programas de reeducação alimentar e de controle do estresse, como também promovam a adoção de hábitos saudáveis de práticas de atividades físicas com os próprios professores de Educação Física.

As evidências encontradas sobre o nível de associação entre os construtos dos parâmetros individual e sócio-ambiental das condições de vida do trabalhador docente em Educação Física foram consideradas insuficientes para indicar relação entre a qualidade de vida no trabalho e o estilo de vida. Mesmo apresentando valores percentuais similares de percepção e comportamentos globais em ambos os construtos, o índice de correlação foi classificado como muito fraco.

$\mathrm{Na}$ análise pormenorizada das matrizes analíticas, constatou-se que o parâmetro sócioambiental qualidade de vida no trabalho evidenciou maior associação entre as suas dimensões. Estas evidências são esperadas, pelo fato de que as dimensões que compõem o construto do trabalho possuem maior ligação entre elas, por tratar sobre um tema mais especifico.

Entretanto, na avaliação do parâmetro individual, estilo de vida, observou-se que os componentes possuem uma característica mais independente $\mathrm{e}$ abrangente da vida do trabalhador docente. Por este fato, constatouse a fraca associação entre os componentes que compõem esta matriz analítica. Contudo, vale destacar que as avaliações globais, tanto do estilo de vida, quanto da qualidade de vida no trabalho, demonstraram os maiores índices de correlação entre os componentes e dimensões das suas respectivas matrizes analíticas.

Nesta perspectiva, evidência-se que tais variáveis avaliam diferentes parâmetros (individual e sócio-ambiental). Assim, salientase que o desprendimento de tempo com o trabalho do professor é grande, tanto no ambiente escolar, quanto nos momentos em que o professor está em seu domicilio preparando e organizado as atividades 
pedagógicas. E, os comportamentos efetuados dentro e fora do ambiente laboral podem interferir diretamente no estilo de vida individual. Observa-se que a análise dos dois construtos reflete temas abrangentes na vida do profissional do magistério, as quais não estão relacionadas entre si diretamente,o que leva a crer que a união destas duas variáveis (qualidade de vida no trabalho e estilo de vida) pode refletir as condições de vida do trabalhador docente.

A continuação das investigações sobre esta temática é sugerida para analisar outros indicadores dos parâmetros individual e sócioambiental das condições de vida do trabalhador docente, bem como possam aprofundar os efeitos mediadores e moderadores de outras variáveis sócio-demográficas e profissionais, como por exemplo: os ciclos vitais, os ciclos de desenvolvimento profissional, o gênero, o estado civil, a formação acadêmica, o tempo de serviço, o pluriemprego, a carga horária de trabalho semanal e o vinculo empregatício.

\section{REFERÊNCIAS}

Al-Mohannadi, A., \& Capel, S. (2007). Stress in physical education teachers in Qatar. Social Psychology of Education, 10, 55-75.

Andrews, J. C. (1993). O stress nos professores de Educação Física dos nossos dias: Uma perspectiva internacional. Boletim da Sociedade Portuguesa de Educação Física, 7/8, 13-25.

Bauer, J., Stamm, A., Virnich, K., Wissing, K., Müller, U., Wirsching, M., \& Schaarschmidt, U. (2006). Correlation between burnout syndrome and psychological and psychosomatic symptoms among teachers. International Archives of Occupational and Environmental Health, 79, 199204.

Blair, S. N., Callingwood, T. R., Reynolds, R., Smith, M., Hagan, D., \& Sterling, C. L. (1984). Health promotion for educators: Impact on health behaviors, satisfaction, and general well-being. American Journal of Public Health, 74 (2), 147-149.

Both, J., \& Nascimento, J. V. (2009). Intervenção profissional na Educação Física escolar: Considerações sobre o trabalho docente. Movimento, 15(2), 169-186.
Both, J., Borgatto, A. F., Nascimento, J. V., Sonoo, C. N., Lemos, C. A. F., \& Nahas, M. V. (2008). Validação da escala "Perfil do Estilo de Vida Individual”. Revista Brasileira de Atividade Física e Saúde, 13(1), 5-14.

Both, J., Nascimento, J. V., Lemos, C. A. F., Donegá, A. L., Ramos, M. H. K. P., Petroski, E. L., \& Duarte, M. F. S. (2006). Qualidade de vida no trabalho percebida por professores de Educação Física. Revista Brasileira de Cineantropometria $e$ Desempenho Humano, 8(2), 45-52.

Bouchard, C., Shephard, R. J., Stephens, T., Sutton, J. R., \& McPherson, B. D. (1990). Exercise, fitness and health: The consensus statement. In C. Bouchard, R. J. Shephard, T. Stephens, J. R. Sutton, \& B. D. McPherson (Eds.), Exercise, fitness and health: A consensus of current knowledge (pp. 3-28). Champaign, Il: Human Kinetics.

Brouwers, A. H., \& Tomic, W. (2000). A longitudinal study of teacher burnout and perceived self-efficacy in classroom management. Teaching and Teacher Education, 16, 239-253.

Carlotto, M. S. (2002). A síndrome de burnout e o trabalho docente. Revista Psicologia em Estudo, 7(1), 21-29.

Carlotto, M. S. (2004). Síndrome de burnout e características de cargo em professores universitários. Revista Psicologia: Organização e Trabalho, 4(2), 145-162.

Dorman, J. P. (2003). Relationship between school and classroom environment and teacher burnout: A LISREL analysis. Social Psychology of Education, 6, 107-127.

Fejgin, N., Talmor, R., \& Erlich, I. (2005). Inclusion and burnout in Physical Education. European Physical Education Review, 11(1), 29-50.

Gasparini, S. M., Barreto, S. M., \& Assunção, A. A. (2005). O professor, as condições de trabalho e os efeitos sobre sua saúde. Educação e Pesquisa, 31 (2), 189-199.

Hardman, K., \& Marshall, J. (2000). The state and status of Physical Education in schools in international context. European Physical Education Review, 6(3), 203-229.

Heyward, V. H. (1998). Advanced fitness assessment and exercise prescription ( $3^{\mathrm{a}}$ ed.). Champaign, Il: Human Kinetics.

Jabnoum, N., \& Fook, C. Y. (2001). Job satisfaction of secondary school teachers in Selangor, Malaysia. International Journal of Commerce $e$ Management, 11(3/4), 72-90. 
Jacas, I. S., Castillo, Z. Y. F., Simón, M. V. S., \& Miclín, O. T. (2000). Afecciones laríngeas en trabajadores de la enseñanza. Revista Cubana de Higiene y Epidemiología, 38(1), 37-42.

Koustelios, A. (2001). Personal characteristics and job satisfaction of Greek teachers. The International Journal of Educational Management, 15(7), 354-358.

Koustelios, A. (2005). Physical Education teachers in Greece: Are they satisfied? International Journal of Physical Education, 42(2), 85-90.

Koustelios, A., \& Tsigilis, N. (2005). The relationship between burnout and job satisfaction among physical education teachers: A multivariate approach. European Physical Education Review, 11 (2), 189-203.

Lapo, F. R., \& Bueno, B. O. (2003). Professores, desencanto com a profissão e abandono do magistério. Cadernos de Pesquisa, 118, 65-88.

Lemos, C. A. F. (2007). Qualidade de vida na carreira profissional de professores de Educação Física do magistério público estadual/RS. Dissertação de Pós-Graduação em Educação Física, Centro de Desportos, Universidade Federal de Santa Catarina, Florianópolis, Brasil.

Lemos, C. A. F., Nascimento, J. V., \& Borgatto, A. F. (2007). Parâmetros individuais e sócioambientais da qualidade de vida percebida na carreira docente em Educação Física. Revista Brasileira de Educação Física e Esporte, 21 (2), 8193.

MacDonald, D. (1995). The role of proletarianization in Physical Education teacher attrition. Research Quarterly for Exercise and Sport, 66 (2), 129-141.

Marques, A. L., Borges, R. S. G., \& Adorno, R. D. (2008). A LDB/96 e a qualidade de vida no trabalho: Com a palavra os docentes da rede pública de Belo Horizonte. Revista de Ciências da Administração, 10(20), 72-94.

Mitra, A., \& Lankford, S. (1999) Research methods in park, recreation and leisure services. Champaign: Sagamore Publishing.

Molina Neto, V. (1998). A prática dos professores de Educação Física nas escolas públicas de Porto Alegre. Movimento, 9(5), 31-46.

Nahas, M. V. (2003). Atividade física, saúde e qualidade de vida: Conceitos e sugestôes para um estilo de vida ativo ( $3^{\mathrm{a}}$ ed.). Londrina: Midiograf.

Nahas, M. V., Barros, M. G. V., \& Francalacci, V. (2000). O pentáculo do bem estar: Base conceitual para a avaliação do estilo de vida em indivíduos ou grupos. Revista Brasileira de Atividade Física e Saúde, 5(2), 48-59.

Nasário, S. T., \& Shigunov, V. (2001). Concepção da prática pedagógica do professor de Educação Física: Importância e influência do aluno. In V. Shigunov, A., \& Shigunov Neto (Eds.), A formação profissional e a prática pedagógica: Ênfase nos professores de Educação Física (pp. 97-119). Londrina: Midiograf.

Nilan, P. (2003). Teachers ' work and schooling in Bali. International Review of Education, 49(6), 563-584.

Pillay, H., Goddard, R., \& Wilss, L. (2005). Wellbeing, burnout and competence: Implications for teachers. Australian Journal of Teacher Education, 30(2), 22-33.

Porto, L. A., Carvalho, F. M., Oliveira, N. F., Silvany Neto, A. M., Araújo, T. M., Reis, E. J. F., \& Delcor, N. S. (2006). Associação entre distúrbios psíquicos e aspectos psicossociais do trabalho de professores. Revista de Saúde Pública, 40(5), 818-826.

Reis, E. J. F., Araújo, T. M., Carvalho, F. M., Barbalho, L., \& Silva, M. O. (2006). Docência e exaustão emocional. Educação e Sociedade, 27(94), 229-253.

Reis, E. J. F., Carvalho, F. M., Araújo, T. M., Porto, L. A., \& Silvany Neto, A. M. (2005). Trabalho e distúrbios psíquicos em professores da rede municipal de Vitória da Conquista, Bahia, Brasil. Cadernos de Saúde Pública, 21(5), 14801490.

Santini, J., \& Molina Neto, V. (2005). A síndrome do esgotamento profissional em professores de Educação Física: Um estudo na rede municipal de ensino de Porto Alegre. Revista Brasileira de Educação Física e Esportes, 19(3), 209-222.

Smith, D., \& Leng, G. W. (2003). Prevalence and sources of burnout in Singapore secondary school Physical Education teachers. Journal of Teaching in Physical Education, 22, 203-218.

Unterbrink, T., Hack, A., Pfeifer, R., BuhlGrießhaber, V, Müller, U., Wesche, H., et al. (2007). Burnout and effort-reward-imbalance in a sample of 949 German teachers. International Archives of Occupational and Environmental Health, 80, 433-441.

Vedovato, T. G., \& Monteiro, M. I. (2008). Perfil sociodemográfico e condições de saúde e trabalho dos professores de nove escolas estaduais paulistas. Revista da Escola de Enfermagem da USP, 42(2), 290-297. 
Viera, A. O., Hernandez, P. A., \& Fernández, I. (2007). Estrés laboral y el síndrome de burnout en docentes venezolanos. Salud de los Trabajadores, 15(2), 71-88.
Walton, R. E. (1973). Quality of working life: What is it? Sloan Management Review, 15(1), 11-21.

Walton, R. E. (1974). Improving the quality of work life. Harvard Business Review, 18(3), 12-16. 